\title{
Simultaneous QCD analysis for identified and unidentified light charged hadrons
}

\section{Soleymaninia, ${ }^{a, *}$ M. Goharipour, ${ }^{a}$ H. Khanpou ${ }^{b}$ and H. Spiesberger ${ }^{c}$}

${ }^{a}$ School of Particles and Accelerators, Institute for Research in Fundamental Sciences (IPM), P.O.Box 19395-5531, Tehran, Iran

${ }^{b}$ Department of Physics, University of Science and Technology of Mazandaran, P.O.Box 48518-78195, Behshahr, Iran

${ }^{c}$ PRISMA ${ }^{+}$Cluster of Excellence, Institut für Physik, Johannes-Gutenberg-Universität, Staudinger Weg 7, D-55099 Mainz, Germany

E-mail: Maryam_Soleymaninia@ipm.ir, Muhammad.Goharipour@ipm.ir, HKhanpour@mazust.ac.ir, spiesber@uni-mainz.de

In this paper, we determine the precise fragmentation functions (FFs) of charged pions $\left(\pi^{ \pm}\right)$, charged kaons $\left(K^{ \pm}\right)$, proton/antiprotons $(p / \bar{p})$ as well as unidentified light charged hadrons $\left(h^{ \pm}\right)$. This determination is performed through a simultaneous QCD analysis of all available experimental data from single-inclusive hadron production in electron-positron annihilation (SIA) processes for $\pi^{ \pm}, K^{ \pm}, p / \bar{p}$ and $h^{ \pm}$production. Our results show that these data have significant impact on both size and uncertainties of the FFs. We apply higher-order perturbative QCD corrections up to next-to-next-to-leading order (NNLO) and also include finite-hadron mass effects.

*** The European Physical Society Conference on High Energy Physics (EPS-HEP2021), ***

*** 26-30 July 2021 ***

*** Online conference, jointly organized by Universität Hamburg and the research center DESY ***

\footnotetext{
${ }^{*}$ Speaker
} 


\section{Introduction}

There have been several QCD analyses to extract the fragmentation functions (FFs) of pions, kaons, protons, and unidentified light charged hadrons individually [1-8], in which experimental data from different processes have been used, such as single inclusive hadron production in electron-positron annihilation (SIA), semi-inclusive deep inelastic scattering (SIDIS), and from proton-(anti)proton collisions. However, they are different in other aspects, such as the phenomenological framework, the QCD perturbative order and the error calculation procedure. The main goal of the present study is to develop our previous analysis [9] to calculate the FFs for charged pions $\left(\pi^{ \pm}\right)$, charged kaons $\left(K^{ \pm}\right)$, proton/antiproton $(P / \overline{(P)})$ and unidentified light charged hadrons $\left(h^{ \pm}\right)$ simultaneously by using all SIA data for these hadrons production. Our results show this simultaneous QCD analysis along with considering hadron mass corrections up to next-to-next-to-leading order (NNLO) accuracy, improves the fit quality significantly and leads to well-constrained FFs.

\section{The QCD framework}

Unidentified light charged hadrons include $\pi^{ \pm}, K^{ \pm}, p / \bar{p}$, and residual light charged hadrons whose contributions are small in comparison with pion, kaon and proton. Hence, the FFs of unidentified light charged hadrons are given by

$$
D_{i}^{h^{ \pm}}=D_{i}^{\pi^{ \pm}}+D_{i}^{K^{ \pm}}+D_{i}^{p / \bar{p}}+D_{i}^{r e s^{ \pm}} .
$$

We parametrize the pion, kaon and proton FFs at the initial scale $Q_{0}=5 \mathrm{GeV}$ by the following functional form [10]:

$$
D_{i}^{\pi^{ \pm}, K^{ \pm}, p / \bar{p}}\left(z, Q_{0}\right)=\frac{\mathcal{N}_{i} z^{\alpha_{i}}(1-z)^{\beta_{i}}\left[1+\gamma_{i}(1-z)^{\delta_{i}}\right]}{B\left[2+\alpha_{i}, \beta_{i}+1\right]+\gamma_{i} B\left[2+\alpha_{i}, \beta_{i}+\delta_{i}+1\right]},
$$

where $i=u^{+}, d^{+}, s^{+}, c^{+}, b^{+}$and gluon $g$, with $q^{+}=q+\bar{q}$. For pion FFs, we assume isospin symmetry $D_{u^{+}}^{\pi^{ \pm}}=D_{d^{+}}^{\pi^{ \pm}}$. However for kaon, we allow all light flavor FFs to be different $D_{u^{+}}^{K^{ \pm}} \neq D_{d^{+}}^{K^{ \pm}} \neq D_{s^{+}}^{K^{ \pm}}$. We consider a relation between $u^{+}$and $d^{+}$FFs for proton by a $z$ independent normalization factor $D_{u^{+}}^{p / \bar{p}}=\mathcal{N} D_{d^{+}}^{p / \bar{p}}$.

The residual light hadrons contribution is rather small, and we consider a simple parametrization function for the residual light charged hadrons FFs given by

$$
D_{i}^{\mathrm{res}^{ \pm}}\left(z, Q_{0}\right)=\mathcal{N}_{i} \frac{z^{\alpha_{i}}(1-z)^{\beta_{i}}}{B\left[2+\alpha_{i}, \beta_{i}+1\right]},
$$

where $i$ refers to $d^{+}, u^{+}, s^{+}, c^{+}, b^{+}$, and $g$. The $S U(3)$ flavor symmetry of light quark flavors is applied for residual hadron FFs, $D_{u^{+}}^{\text {res }^{ \pm}}=D_{d^{+}}^{\text {res }^{ \pm}}=D_{s^{+}}^{\text {res }^{ \pm}}$.

\section{Results and discussions}

In this section, we systematically study our analysis and the additional constraints due to the inclusion of unidentified charged hadron experimental data. For this purpose, we first determine 
the pion, kaon, and proton FFs by performing three separate analyses of the related SIA data as usual. Then we include also the unidentified light charged hadron measurements and perform a simultaneous analysis of all data to study how it affects the extracted FFs. The results obtained for the $\pi^{ \pm}, K^{ \pm}$and $p / \bar{p}$ FFs from these two approaches at the scale $Q^{2}=100 \mathrm{GeV}^{2}$ are compared in Figs. 1, 2 and 3, respectively, where all FFs have been normalized to their central values calculated in the separate hadron fits.

As can clearly be seen from Figs. 1, 2 and 3, the inclusion of unidentified light hadron data modifies the central values and the uncertainties of FFs. In the case of the charged pion FF in Fig. 1, the reduction of the uncertainty bands is noticeable, especially for $D_{g}^{\pi^{ \pm}}$and $D_{\Sigma}^{\pi^{ \pm}}$. Also, one can see the effect of including the unidentified light charged hadron data on the shape of gluon FF of pion. In Fig. 2, similarly, we can see the reduction of the uncertainties for $D_{g}^{K^{ \pm}}$and $D_{\Sigma}^{K^{ \pm}}$, but by a smaller factor in comparison to pion FFs. In the final case in Fig. 3 remarkable changes of the shapes can be seen for gluon and heavy quark FFs. Here again, a large decrease in the uncertainty bands is visible for all FFs except for the $\Sigma$ FF.

We present here, in the last four columns of Table 1, the values of $\chi^{2}$ per number of data points, $\chi^{2} /\left(N_{\text {pts. }}\right)$, for each data set individually. The value of the total $\chi^{2}$ per number of degrees of freedom, $\chi^{2} /($ d.o.f.), is shown in the last row of this table. It should be noted that the final number of data points of each experimental data set shown in the tables is subject to kinematic cuts. After imposing the kinematical cuts, our total number of data is $N_{\text {pts. }}=1492$. In addition, we find $\chi^{2} /$ d.o.f. $=1.171$ for NLO and $\chi^{2} /$ d.o.f. $=1.083$ for NNLO fits and so the NNLO fit shows in general a better fit quality.
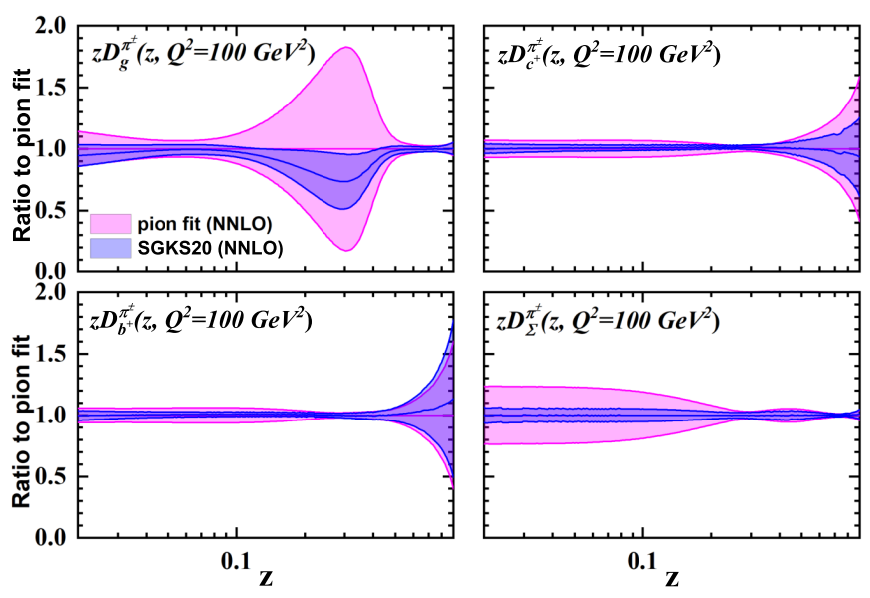

Figure 1: Comparison of SGKS20 NNLO charged pion FFs, $z D_{i}^{\pi^{ \pm}}\left(z, Q^{2}=100 \mathrm{GeV}^{2}\right)(i=g, c, b, \Sigma)$ obtained from a simultaneous analysis of all $\pi^{ \pm}, K^{ \pm}, p / \bar{p}$ and $h^{ \pm}$data with corresponding ones obtained from the analysis of $\pi^{ \pm}$data separately. 

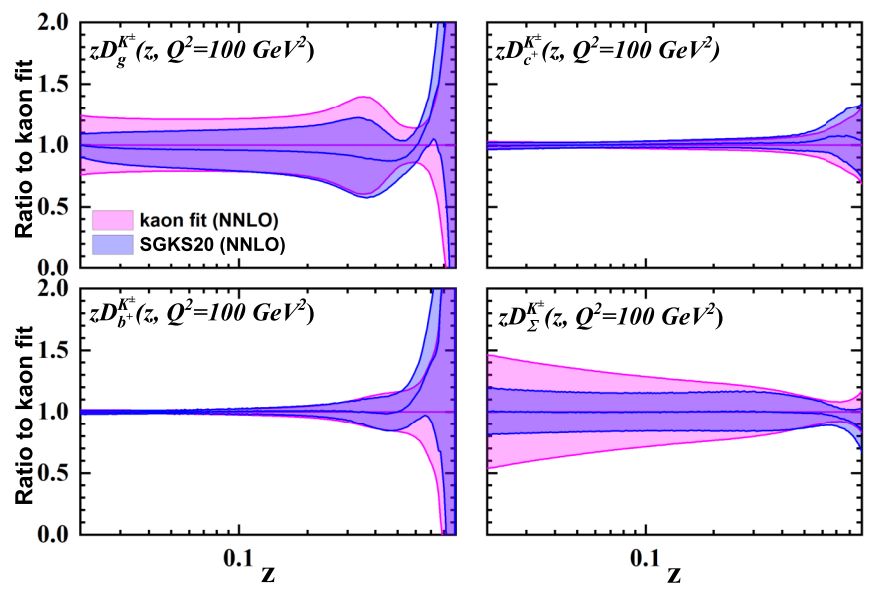

Figure 2: Same as Fig. 1, but for the charged kaon $z D_{i}^{K^{ \pm}}\left(z, Q^{2}\right)$ FFs.
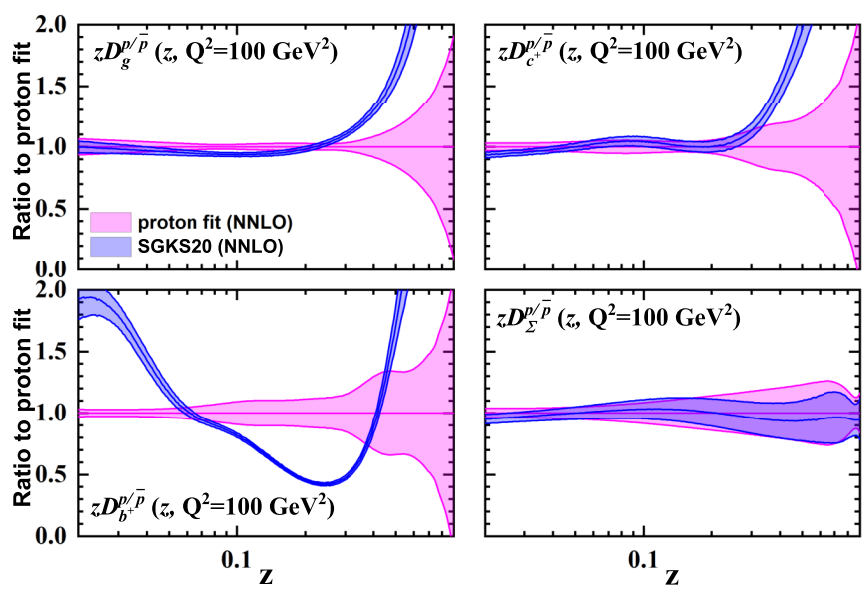

Figure 3: Same as Fig. 1, but for the proton and antiproton FFs, $z D_{i}^{p / \bar{p}}\left(z, Q^{2}\right)$.

\section{References}

[1] R. A. Khalek, V. Bertone and E. R. Nocera, Phys. Rev. D 104 (2021) no.3, 034007 doi:10.1103/PhysRevD.104.034007 [arXiv:2105.08725 [hep-ph]].

[2] E. Moffat et al. [Jefferson Lab Angular Momentum (JAM)], Phys. Rev. D 104, no.1, 016015 (2021) doi:10.1103/PhysRevD.104.016015 [arXiv:2101.04664 [hep-ph]]. 
[3] V. Bertone et al. [NNPDF Collaboration], Eur. Phys. J. C 78, 651 (2018), [arXiv:1807.03310 [hep-ph]].

[4] M. Soleymaninia, M. Goharipour and H. Khanpour, Phys. Rev. D 98, 074002 (2018), [arXiv:1805.04847 [hep-ph]].

[5] V. Bertone et al. [NNPDF Collaboration], Eur. Phys. J. C 77, 516 (2017), [arXiv:1706.07049 [hep-ph]].

[6] M. Epele, C. García Canal and R. Sassot, Phys. Lett. B 790, 102 (2019), [arXiv:1807.07495 [hep-ph]].

[7] D. de Florian, R. Sassot, M. Epele, R. J. Hernández-Pinto and M. Stratmann, Phys. Rev. D 91, 014035 (2015), [arXiv:1410.6027 [hep-ph]].

[8] D. de Florian, M. Epele, R. J. Hernández-Pinto, R. Sassot and M. Stratmann, Phys. Rev. D 95, 094019 (2017), [arXiv:1702.06353 [hep-ph]].

[9] M. Soleymaninia, M. Goharipour and H. Khanpour, Phys. Rev. D 99, 034024 (2019), [arXiv:1901.01120 [hep-ph]].

[10] M. Soleymaninia, M. Goharipour, H. Khanpour and H. Spiesberger, Phys. Rev. D 103, no.5, 054045 (2021) doi:10.1103/PhysRevD.103.054045 [arXiv:2008.05342 [hep-ph]].

[11] M. Leitgab et al. [Belle Collaboration], Phys. Rev. Lett. 111, 062002 (2013), [arXiv:1301.6183 [hep-ex]].

[12] J. P. Lees et al. [BaBar Collaboration], Phys. Rev. D 88, 032011 (2013), [arXiv:1306.2895 [hep-ex]].

[13] R. Brandelik et al. [TASSO Collaboration], Phys. Lett. 94B, 444 (1980).

[14] W. Braunschweig et al. [TASSO Collaboration], Z. Phys. C 47, 187 (1990).

[15] H. Aihara et al. [TPC/Two Gamma Collaboration], Phys. Rev. Lett. 61, 1263 (1988).

[16] W. Braunschweig et al. [TASSO Collaboration], 5"Pion, Kaon and Proton Cross-sections in $e^{+} e^{-}$Z. Phys. C 42, 189 (1989).

[17] D. Buskulic et al. [ALEPH Collaboration], Z. Phys. C 66, 355 (1995).

[18] D. Buskulic et al. [ALEPH Collaboration], Phys. Lett. B 357, 487 (1995), Erratum: [Phys. Lett. B 364, 247 (1995)].

[19] P. Abreu et al. [DELPHI Collaboration], Eur. Phys. J. C 5, 585 (1998).

[20] R. Akers et al. [OPAL Collaboration], Z. Phys. C 63, 181 (1994).

[21] K. Ackerstaff et al. [OPAL Collaboration], Eur. Phys. J. C 7, 369 (1999), [hep-ex/9807004].

[22] K. Abe et al. [SLD Collaboration], Phys. Rev. D 69, 072003 (2004), [hep-ex/0310017].

[23] M. Althoff et al. [TASSO Collaboration], Z. Phys. C 17, 5 (1983). 


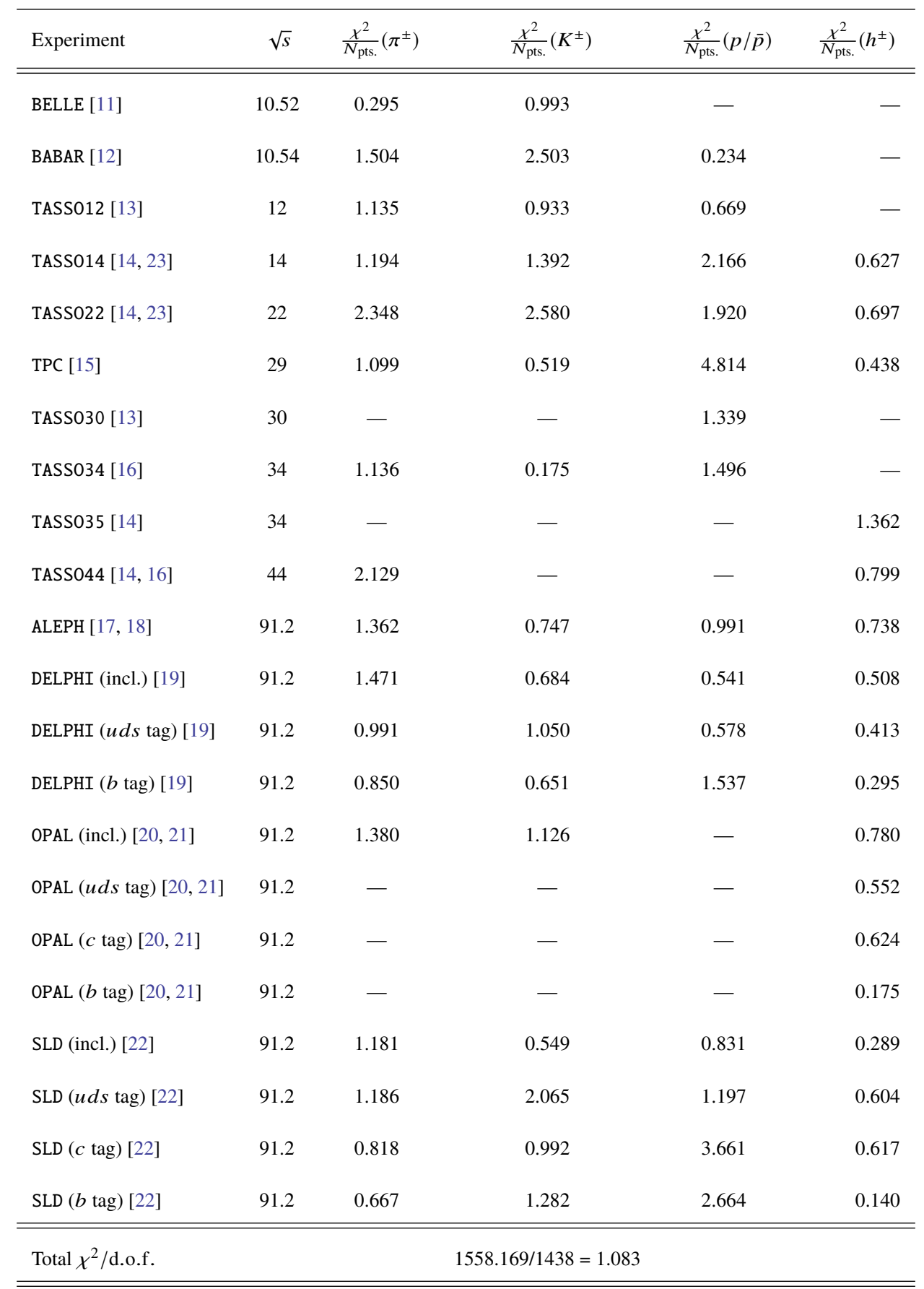

Table 1: The list of data sets for $\pi^{ \pm}, K^{ \pm}, p / \bar{p}$, and $h^{ \pm}$production used in the present analysis. For each input data set, we have indicated the corresponding reference and the center-of-mass energy. In the last four columns the value of $\chi^{2} / N_{\text {pts. }}$ resulting from the FF fit are shown at NNLO order. We show the total value of $\chi^{2} /$ d.o.f. at the bottom of the table. 